\title{
Solar-Neutrino Detection Gets a Boost
}

\author{
A new measurement method allows researchers to obtain directional \\ information about low-energy solar neutrinos, something that was \\ previously hard to do.
}

\author{
By Katie McCormick
}

$\square$ cientists monitor solar neutrinos, produced by nuclear fusion in the Sun, to gain insights into the processes happening in the Sun's core. To detect solar neutrinos, physicists typically use two types of detector, one that has directional sensitivity-it can tell where the neutrinos came from-and another that has better energy sensitivity-it can register lower-energy neutrinos. Now, by combining the strengths of these two detectors, the team behind the Borexino detector has shown that Borexino can have both capabilities [1, 2]. Their measurements indicate that Borexino can record both the energies and the paths of sub-1-MeV neutrinos.

The two most common types of solar-neutrino detectors are the water Cherenkov detector and the liquid scintillator detector. Water Cherenkov detectors pick up neutrinos through the electromagnetic Cherenkov radiation from their interaction

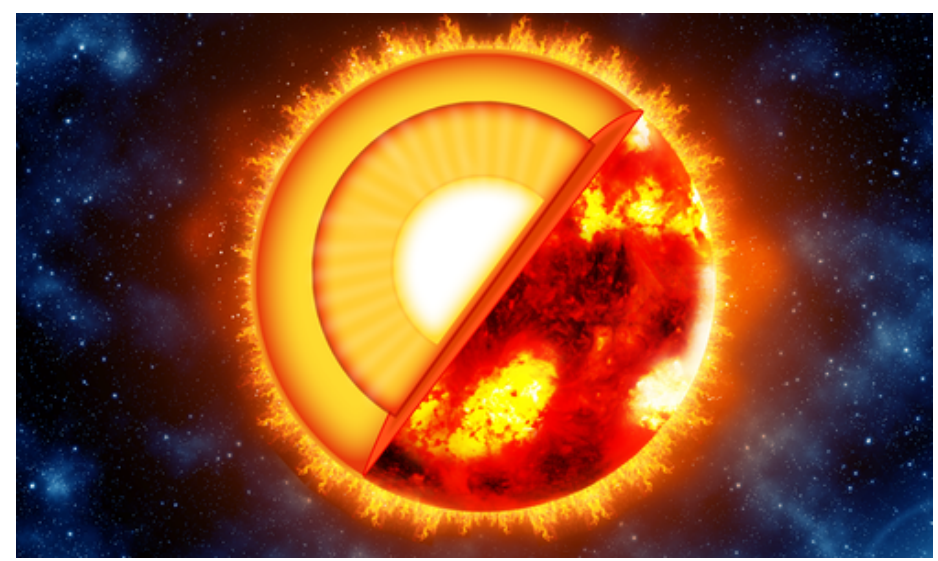

Credit: vadarshop/stock.adobe.com with electrons in water. The properties of this radiation are direction-dependent, making the technique excellent for reconstructing neutrino paths. But water Cherenkov detectors typically have low sensitivity to MeV neutrinos, making them useless for studying low-energy neutrinos. Liquid scintillator detectors spot similar neutrino emissions, but they use an oil doped with a scintillator. That doping results in a much lower energy cutoff-about $0.2 \mathrm{MeV}$. But this type of detector isn't sensitive to the directional information of the neutrinos, something the Borexino Collaboration has now solved.

The team developed a method for analyzing data from a liquid scintillator that allows them to tease out the directional dependence of the neutrino signal. The method involves comparing the cumulative distribution of the detected radiation from many neutrinos to that of a simulation.

The Borexino team plans to use their method to study in greater detail low-energy solar neutrinos. They also hope to learn more about the fundamental nature of neutrinos, a mystery that still eludes particle physicists.

Katie McCormick is a freelance science writer based in Seattle, WA.

\section{REFERENCES}

1. M. Agostini et al. (Borexino Collaboration), "First directional measurement of sub-MeV solar neutrinos with Borexino," Phys. Rev. Lett. 128, 091803 (2022).

2. M. Agostini et al. (Borexino Collaboration), "Correlated and integrated directionality for sub-MeV solar neutrinos in Borexino," Phys. Rev. D 105, 052002 (2022). 